\title{
Reversible Lower Motor Neuron Disease: A New Case of a Forgotten Disease
}

\author{
Thiago Cardoso Vale, ${ }^{\mathrm{a}, \mathrm{b}}$, Denise da Silva Freitas ${ }^{\mathrm{a}}$, Luiz Sergio Mageste ${ }^{\mathrm{a}}$, \\ Leonardo Dornas de Oliveira ${ }^{\mathrm{a}}$, Antonio Lucio Teixeira ${ }^{\mathrm{a}}$
}

\begin{abstract}
Motor neuron disease (MND) is mostly associated with an irreversible course. Spontaneous recovery has been rarely reported. Herein described is a case of a spontaneous recovery of a lower motor neuron disease. A 38-year-old man complained of an insidious onset of weakness in the right upper limb that progressed to the lower limbs in 16 months. Physical examination revealed mild dysphonia, dysphagia, fasciculations, global hypotonia without prominent atrophy, proximal and distal tetraparesis. The patient was wheelchair-bound. EMG revealed signs of recent and chronic denervation involving bulbar, axial and appendicular myotomes with abundant positive sharp waves and fibrillations potentials in all muscles tested. In two year follow-up, the patient evolved with complete recovery and a new EMG study was completely normal. Reversible MND is a condition rarely reported, but physicians should keep in mind the possibility of its occurrence.
\end{abstract}

Keywords: Motor neuron disease; Anterior horn cell disorders; Lower motor neuron disease; Amyotrophic lateral sclerosis

\section{Introduction}

The term 'motor neuron disease' (MND) is mostly associated with an irreversible progression of motor symptoms to death in few years. Several case reports of secondary MND have been published, in which motor symptoms remitted after treatment of the underlying causes, such as lymphoma

Manuscript accepted for publication January 30, 2013

\footnotetext{
${ }^{a}$ Neurology Division, University Hospital, Faculty of Medicine, Federal University of Minas Gerais (UFMG), Belo Horizonte, Minas Gerais, Brazil

${ }^{\mathrm{b} C o r r e s p o n d i n g ~ a u t h o r: ~ T h i a g o ~ C a r d o s o ~ V a l e, ~ S e r v i c o ~ d e ~ N e u r o l o-~}$ gia/Hospital das Clinicas, Universidade Federal de Minas Gerais Avenida Alfredo Balena 110, CEP 30130-100 Belo Horizonte, Minas Gerais, Brasil. Email: thiagocardosovale@hotmail.com
}

doi: http://dx.doi.org/10.4021/jnr171w
[1], carcinoma [2], cervical trauma [3], paraproteinaemia [4] and heavy metal intoxication [5]. Spontaneous remission of non-symptomatic MND is extremely rare.

\section{Case Report}

In May 2008, a 38-year-old man complained of an insidious onset of weakness in the right upper limb. After the progression of the weakness to the lower limbs in one year and four months, he was first evaluated at our clinic. He also presented speech and swallowing difficulties. He denied systemic diseases, sensory symptoms, urinary/faecal sphincter impairment, sexual dysfunction, exposure to toxic agents, tobacco, alcohol or recreational drugs. Physical examination revealed mild dysphonia, dysphagia, fasciculations, global hypotonia with mild atrophy in the proximal and distal paretic muscles (upper-limb strength MRC graded 3 and lower-limb strength MRC graded 2) and brisk reflexes without Hoffmann and Babinski signs. The patient was wheelchair bound.

An extensive work-up revealed absence of abnormalities in brain, cervical and thoracic magnetic resonance imaging, cerebrospinal fluid analysis, blood screen chemistry, ions, platelet count, liver function, creatine kinase, thyroid hormones, serum protein electrophoresis, complement and vitamin levels, HTLV I and II, VDRL and HIV serologies and auto-immune pannel (rheumatoid and antinuclear factors, anticardiolipin, lupus anticoagulant, anti-SSa, anti-SSb, anti-DNA, anti-RNP, anti-Sm antibodies). Twenty-four-hour urine specimen was negative for heavy metals (lead, mercury and arsenic) and blood lead levels were normal. Chest $\mathrm{x}$-rays, abdominal ultrassonography as well as basic laboratory screening (prostate-specific and carcinoembryonic antigens, alpha-fetoprotein) for occult malignancy were also negative. Nerve conduction studies showed normal sensory and motor action potentials, with no conduction block or focal abnormalities. Late responses (F wave) were also normal. Electromyography (EMG) revealed signs of recent and chronic denervation involving bulbar, axial and appendicular myotomes with abundant positive sharp waves and fibrillations potentials in all muscles tested, including tongue, sternocleidomastoid, thoracic paraspinal and limb muscles, with 
decreased recruitment and neurogenic motor unit potentials. The electrophysiological pattern was compatible with inferior MND.

Treatment with Riluzole $50 \mathrm{mg}$ twice a day was started in February 2010, but adverse effects such as gastric intolerance prompted its withdrawal shortly after. At this time, the patient mentioned improvement in swallowing and in a 3 month-follow up consultation he denied any dysphagia. In a 6 month-follow up consultation, he reported gradual improvement of speech and muscle strength, leading to the use of a bilateral support to walk. In November 2011, his muscle strength was normal and he was walking without the need of any help. In February 2012, another electroneuromyography performed by the same neurophysiologist was considered normal. The patient recovered his physical capacity.

\section{Discussion}

Our case illustrates a spontaneous remission of MND, defined by clinical and electroneuromyographic studies. There are further cases described in the literature. Mulder and Howard [6] reported that, during the period of 20 years examining more than 2,000 patients with amyotrophic lateral sclerosis (ALS), they had seen "several" patients who were initially diagnosed with ALS but subsequently recovered. Tucker and Layzer [7] described four patients with a clinical syndrome closely resembling ALS that recovered completely in 5 - 12 months after onset. In 1993, Tsai et al [8] described the latest case of a clinically resembling ALS that recovered completely in approximately 1.5 years without having any underlying systemic disease, malignancy or intoxication. All previously described cases had both superior and inferior MND features, being characterised as ALS-like syndrome, which differs from our case with predominantly inferior MND features. Given the presence of brisk reflexes in a patient with clear muscle denervation, a superior MND should not be ruled out. One might suggest that the diffuse and abundant fibrillations and positive waves without evident muscle atrophy or reduced motor action potentials on EMG are suggestive of muscle membrane dysfunction rather than denervation. However, no myopathic finding was observed and the motor unit potentials had a neurogenic pattern with decreased recruitment. Our case has diagnostic limitations due to lack of tests for channelopathies, assessment by means of motor evoked potentials or muscle biopsy. All the same, unusual features were present: presence at EMG of active denervation without fasciculation, as well as the coexistence of brisk tendon reflexes, a repeated EMG performed after the clinical recovery with normal results without signs of motor unit rearrangement. Reversible MND is an enigmatic rare condition that physicians can eventually meet.

\section{Author Contributions}

Study concept and design: Oliveira, Teixeira. Acquisition of data: Vale, Freitas, Mageste, Oliveira. Analysis and interpretation of data: Vale, Freitas, Mageste, Oliveira, Teixeira. Drafting of the manuscript: Vale, Freitas, Oliveira. Critical revision of the manuscript for important intellectual content: Oliveira, Teixeira. Administrative, technical and material support: Vale, Freitas, Mageste.

\section{Declaration}

We declare no conflicts of interest/no financial support.

\section{References}

1. Younger DS, Rowland LP, Latov N, Hays AP, Lange DJ, Sherman W, Inghirami G, et al. Lymphoma, motor neuron diseases, and amyotrophic lateral sclerosis. Ann Neurol. 1991;29(1):78-86.

2. Evans BK, Fagan C, Arnold T, Dropcho EJ, Oh SJ. Paraneoplastic motor neuron disease and renal cell carcinoma: improvement after nephrectomy. Neurology. 1990;40(6):960-962.

3. Gallagher JP, Sanders M. Trauma and amyotrophic lateral sclerosis: a report of 78 patients. Acta Neurol Scand. 1987;75(2):145-150.

4. Rudnicki S, Chad DA, Drachman DA, Smith TW, Anwer UE, Levitan N. Motor neuron disease and paraproteinemia. Neurology. 1987;37(2):335-337.

5. Boothby JA, DeJesus PV, Rowland LP. Reversible forms of motor neuron disease. Lead "neuritis". Arch Neurol. 1974;31(1):18-23.

6. Mulder DW, Howard FM, Jr. Patient resistance and prognosis in amyotrophic lateral sclerosis. Mayo Clin Proc. 1976;51(9):537-541.

7. Tucker T, Layzer RB, Miller RG, Chad D. Subacute, reversible motor neuron disease. Neurology. 1991;41(10):1541-1544.

8. Tsai CP, Ho HH, Yen DJ, Wang V, Lin KP, Liao KK, Wu ZA. Reversible motor neuron disease. Eur Neurol. 1993;33(5):387-389. 